\title{
Clinical features associated with the development of hydrocephalus following TBI in the paediatric age group
}

\author{
Ronak Ved $^{1}$ (D) $\cdot$ Rebecca Fraser $^{1} \cdot$ Sarah Hamadneh $^{1} \cdot$ Malik Zaben $^{1} \cdot$ Paul Leach $^{1}$
}

Received: 27 October 2019 / Accepted: 22 June 2020 / Published online: 29 June 2020

(C) The Author(s) 2020

\begin{abstract}
Introduction Predictive factors for post-traumatic hydrocephalus (PTH) in adults have been elucidated but remain uncertain for children. We aimed to identify the prevalence of PTH in paediatric patients and identify clinical/radiological factors which may increase the probability of children developing PTH.

Methods This was a retrospective study of all patients $<16$ years old admitted to our unit with traumatic brain injury (TBI) between March 2013 and June 2018, 108 patients in total. Patients were classified as mild (13-15), moderate (9-12) or severe (38) TBI based on admission GCS. Three independent reviewers collected data from case notes. CT scans were reviewed for hydrocephalus using Evan's index. Two-tailed Fisher's exact tests with a $p$ value $<0.05$ were considered statistically significant. Results Median patient age was 7 years, and $65 \%$ were males $(n=70)$. PTH wasn't identified in any patients with mild/moderate TBI $(n=79)$. In cases of severe TBI $(n=29)$, three patients developed PTH requiring ventriculoperitoneal shunting $(10 \% ; p=$ $0.02)$. Radiological features which were significantly more common in the PTH group were intraventricular haemorrhage $(p=$ $0.05)$ and subarachnoid haemorrhage $(p=0.03)$. There was also a trend towards a statistically significant association with subdural haematoma $(p=0.07)$. The need for other neurosurgical procedures, such as fracture elevation and craniotomy, did not increase the probability of developing with PTH $(p=0.08)$.

Discussion The prevalence of PTH in our study is $2.7 \%$. Factors which may be associated with a higher probability of developing PTH may include IVH, SAH, severity of TBI, and subdural haematoma. We propose a national prospective multicentre database of paediatric PTH. The data collected on prevalence, presentation, risk factors, and management could guide contemporary management and improve the outcomes of children with PTH.
\end{abstract}

Keywords Hydrocephalus $\cdot$ Paediatric $\cdot$ Trauma $\cdot$ Injury

\section{Introduction}

Traumatic brain injury (TBI) is a leading cause of death and disability in young people in the world $[1,2]$. Post-traumatic hydrocephalus (PTH) affects $10-40 \%$ of adult patients after severe TBI [3]. It is associated with poor clinical outcomes but is also amenable to surgical treatment, such as CSF shunting [4]. Factors such as injury severity, the presence of subdural haematomas, and the need for surgery have been associated with an increased risk of adult patients developing PTH [5-8].

Ronak Ved

vedr@cardiff.ac.uk

1 Department of Neurosurgery, University Hospital Wales, 4th Floor, Heath Park Way, Cardiff CF14 4XW, UK
Many studies around PTH have assessed adult patients; however, their findings may not necessarily correlate with PTH in the paediatric patient group. There is thus a clinical need to identify the prevalence of PTH in paediatric patients and to assess which factors may predispose patients to the debilitating, but potentially treatable, development of PTH.

\section{Objectives}

The objectives of the present study were to:

1. Ascertain the prevalence of hydrocephalus in paediatric traumatic head injury patients at a single neurosurgical unit

2. Identify potential clinical or radiological features which may predict the development of post-traumatic hydrocephalus in children 


\section{Methods}

Participants were selected via scrutiny of a prospectivelymaintained paediatric TBI database at a single neurosurgical unit. This enabled the study to capture all patients under the age of 16 admitted to a national neurosurgical unit with TBI between March 2013 and June 2018. Electronic and paperbased case notes were then reviewed by three independent investigators. Figure 1 outlines the patient selection process and the data collection processes that were undertaken.

Demographic, clinical, and imaging data at admission, during the hospital stay, and at follow-up were used for this study. All CT scans were reviewed for PTH using the Evan's index and criteria described by Gudeman et al. [2]. For statistical analysis of categorical data, two-tailed Fisher's exact tests with a $p$ value $<0.05$ were considered statistically significant.

\section{Results}

One hundred eight patients were included in the study analysis. The mean age of the cohort was 7 years and 5 months
(11 days-15 years, 9 months). Thirty-five percent of the cohort were female $(n=38)$, and $65 \%(n=70)$ were male. Patient ages were categorized into four age groups: < 2 years, 2-5 years, 6-11 years, and 12-16 years. The most common age bracket for TBI was 6-11 years (Fig. 2). The most common mechanisms of injury were falls and road traffic collisions (Fig. 2). No patients in the study cohort died as a result of their TBI. Median follow-up for the study cohort was 48 months.

TBI patients where next dichotomized (Fig. 3) into a mild and moderate TBI cohort (admission GCS $>8 ; n=79$ ) and a severe TBI cohort (admission GCS 8 or lower; $n=29$ ). No patients in the mild/moderate TBI group developed PTH, whereas three patients who suffered a severe TBI developed PTH (10\%; $p=0.02)$ (Fig. 4).

Since PTH was only seen amongst patients who had suffered serve TBI, this subcohort underwent analysis to identify features which may increase the probability of developing PTH (Table 1). None of the severe TBI patients had evidence of ventriculomegaly on their admission scan. Seventeen of these patients underwent emergency craniotomy, and a further seventeen required other forms of emergency neurosurgery

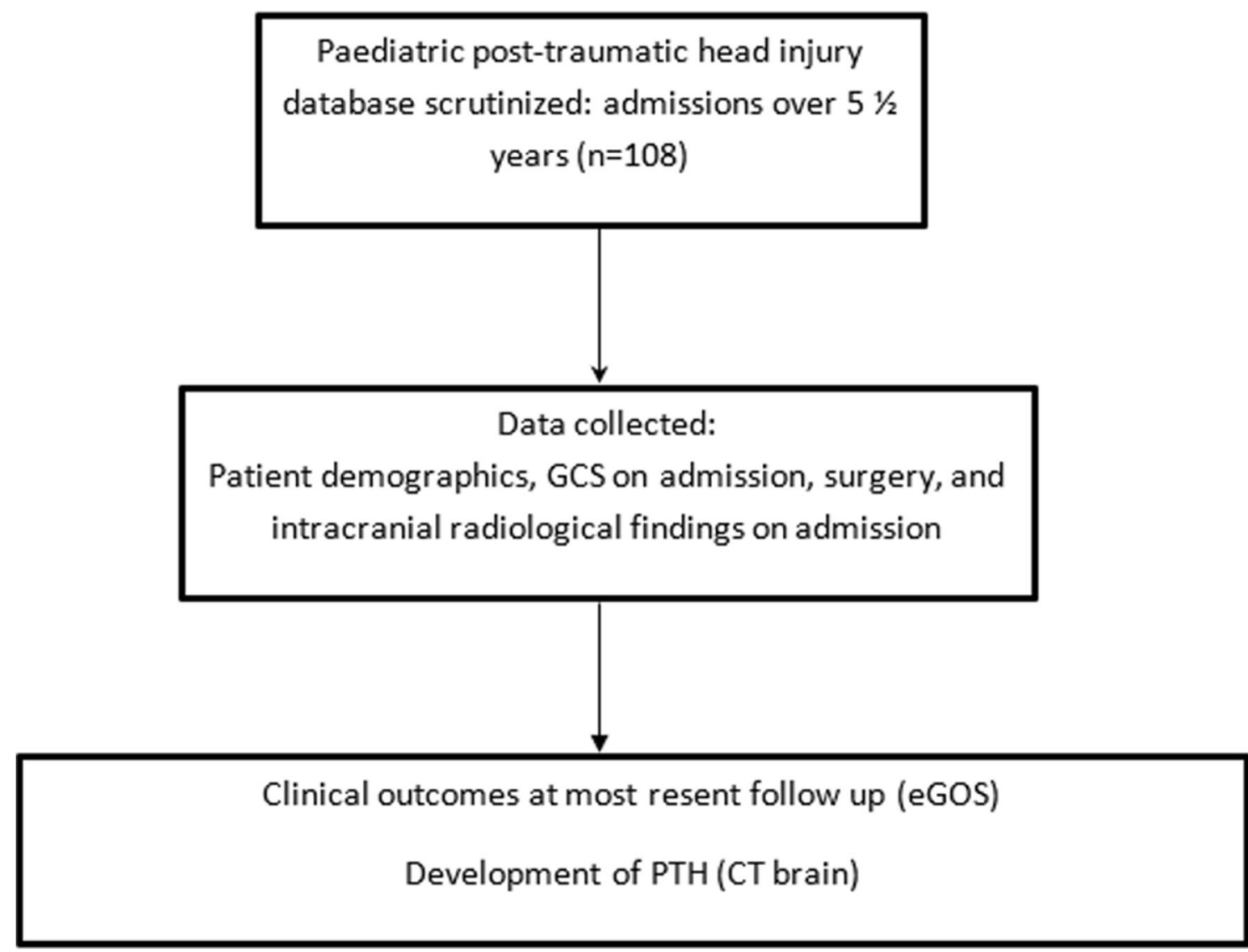

Fig. 1 Method flow chart outlining the participant selection process for the present study. Clinical and radiological findings assessed by the investigators were selected based upon factors listed within the current literature that may predispose adult patients to PTH [5], alongside any other notable features or pathological findings for each patient. The comprehensive list of features assessed for is provided in the results in Table 2 


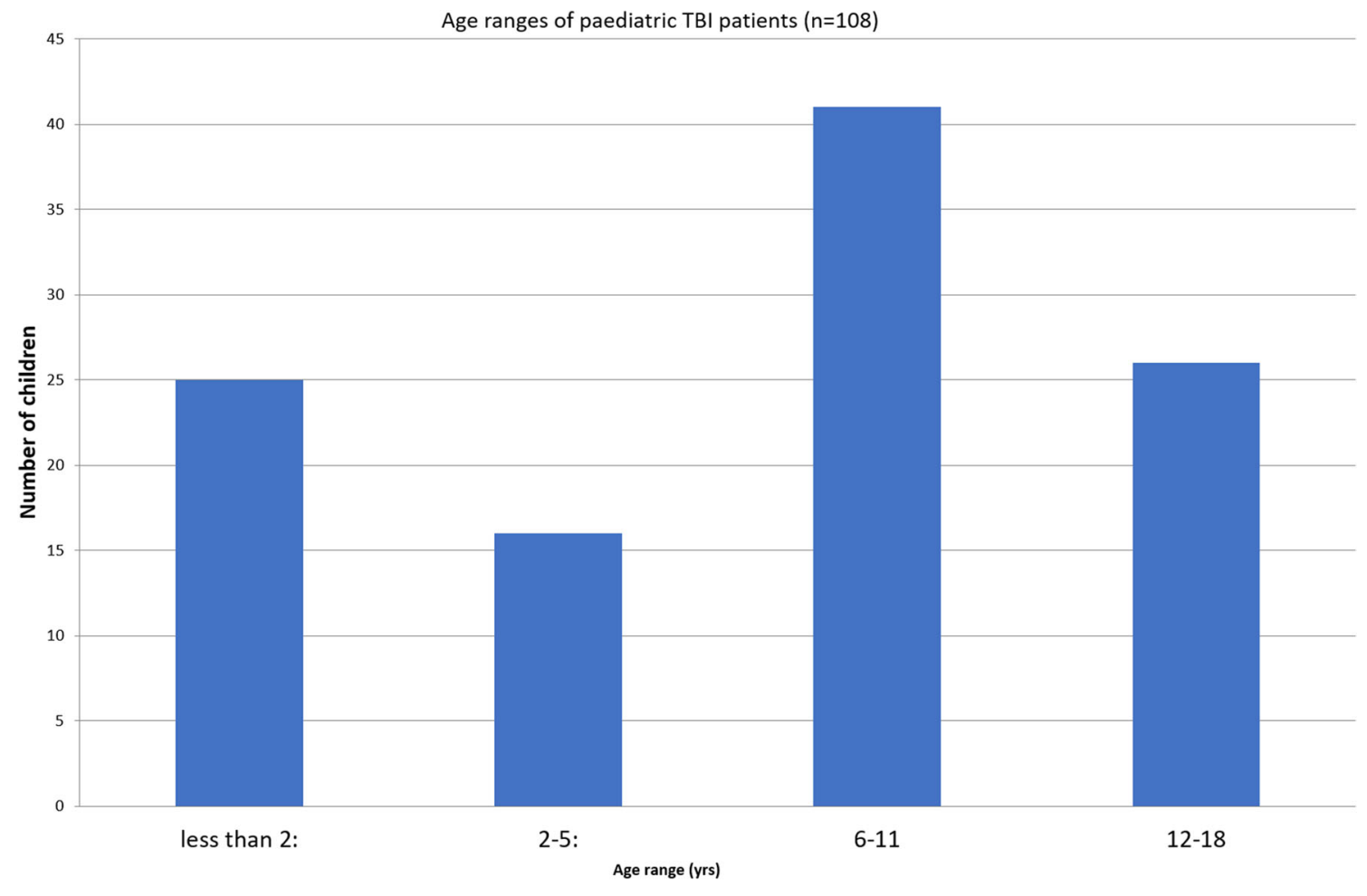

Fig. 2 Age range distribution for TBI across the paediatric cohort. The most common cohort ages for TBI were 6-11 years and 12-18 years

(e.g. elevation of depressed skull fractures, wound washouts, burr hole evacuations of haematomas). One of the patients who underwent emergency neurosurgery (craniotomy for evacuation of a subdural haematoma) went on to develop PTH (Tables 1 and 2). The need for emergency neurosurgery of any kind did not predict the development of PTH $(p=0.43)$.

The radiological features at the time of their injury for all three patients who developed PTH are outlined in more detail in Table 2. IVH and SAH were significantly associated with subsequent PTH, whilst there was also a trend towards significance for association between SDH with the development of PTH (Table 2).

Two of the patients who developed PTH were 9 years old at the time of their injury. One was aged 10 months. Two were female (age 9 years and 10 months), and one was male (age 9 years). The mechanisms of injury were one road traffic collision, one fall from height, and one hit by a large falling object. Whilst all three suffered a skull fracture, none of these were depressed fractures. Only one patient underwent emergency surgery (Table 3). PTH developed between 1 and 3 months post-injury, with presenting symptoms and signs including persistent headaches, slow neurological recovery, and drowsiness, respectively, amongst the three patients. All three patients underwent insertion of VP shunt upon diagnosis of PTH, all achieving satisfactory CSF diversion based on postoperative head scans and improvements in their clinical symptoms. None of the cohort has required revision of their VP shunt up to the date of submission (follow-up periods of 3,
4, and 5 years, respectively; Table 3). Extended Glasgow Outcome Scores ranged from 8 (upper good recovery) to 5 (moderate disability).

\section{Discussion}

PTH is a significant cause of disability in adults after TBI [9]. It may prolong hospital stay, worsen morbidity, and raise hospital costs [10]. Although the characteristics of PTH in adults have been delineated, there is a relative lack of knowledge regarding PTH in children $[2,11]$. The ability to identify children who may be most at risk of developing PTH would be valuable to clinicians for guiding management and prognostication following paediatric TBI. To this end, the present study aimed to identify the prevalence of PTH in a paediatric neurosurgical population and begin to investigate factors which may help to increase the suspicion of clinicians for subsequent development of PTH.

The prevalence of PTH in this study was just under 3\%, rising to $10 \%$ when severe TBI is analysed as a subcohort. These figures are lower than those reported in the literature for adults, which range from 10 to $50 \%[1,5,6,8,12]$. However, the identified prevalence is higher than the reported frequency of PTH in two recent retrospective studies of paediatric $\mathrm{PTH}$, which report prevalence rates of $\sim 1 \%[5,10]$. These large studies are large database reviews of paediatric head injury admissions across multiple healthcare sites. These reviews 


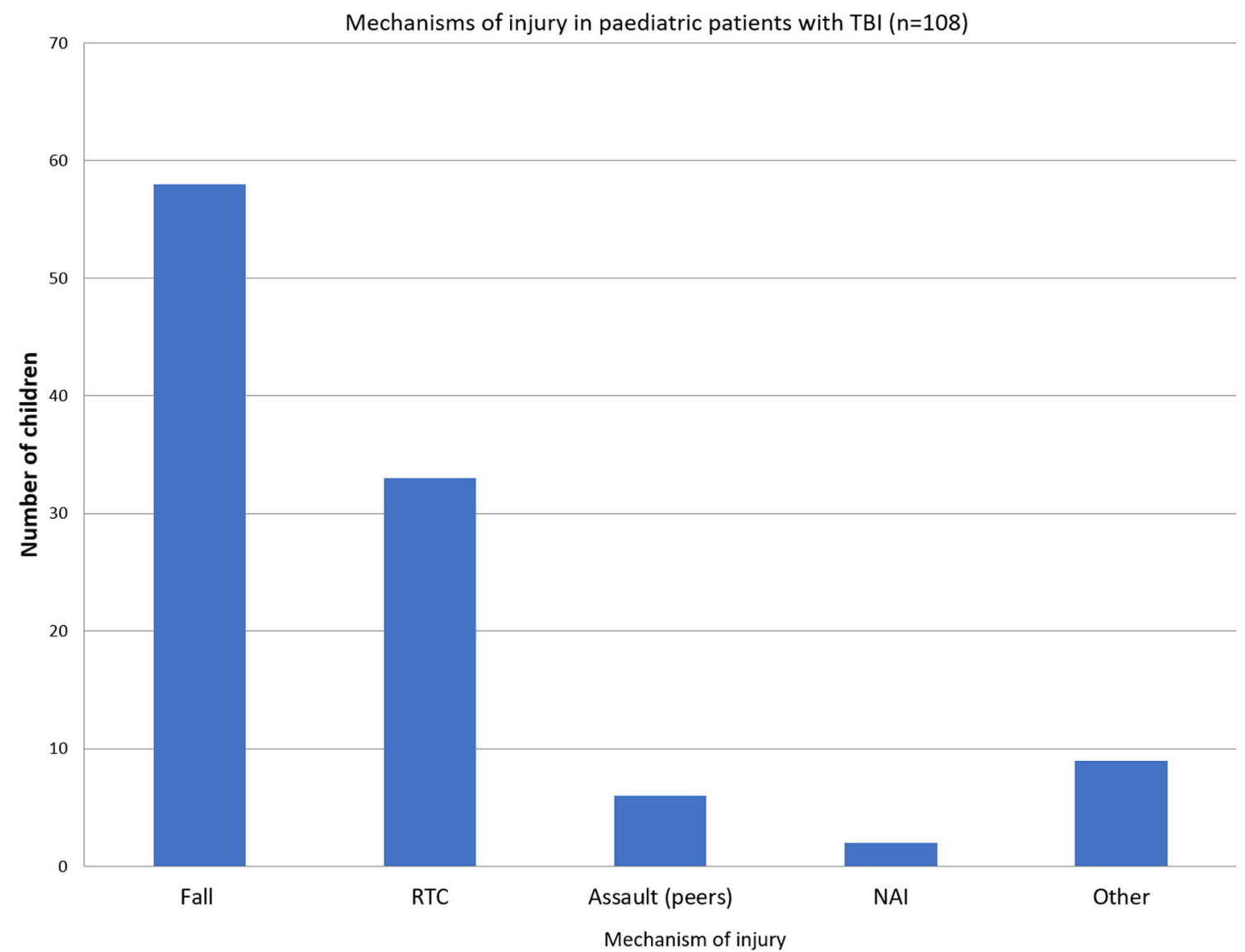

Fig. 3 Mechanism of TBI for paediatric patients. The most common MOIs were falls $(51 \% ; n=55)$ and RTC ( $30 \%$; $n=32)$. 'Other' injuries included objects falling onto children and injuries from animals. RTC road traffic collision, MOI mechanism of injury, NAI non-accidental injury

thus canvassed large numbers of patients, $(\sim 100,000$ each $)$ and reviewed electronic records to identify clinical features which may have associations with PTH. Both studies identified similar prevalence rates of PTH in children and similar clinical features associated with PTH, such as younger age [5] and the need for other neurosurgical procedures [10].

\section{All had VP-shunts}

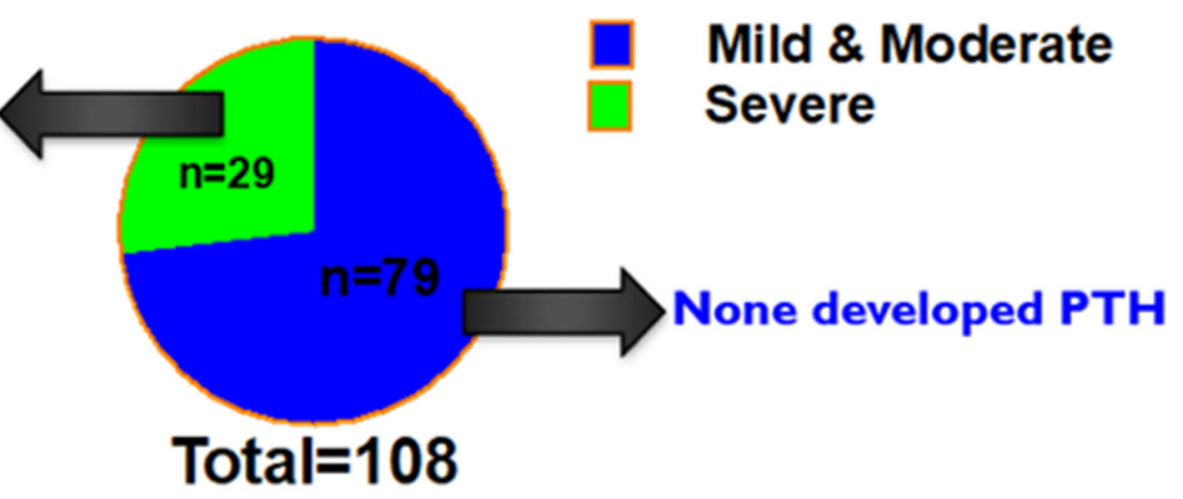

Mild (GCS 13-15) and Moderate

(GCS 9-12) TBI: $n=79$

Severe (GCS 3-8) TBI: $n=29$

Fig. 4 The proportion of paediatric TBI patients presenting with mild and moderate TBI vs those presenting with severe TBI. All patients who developed PTH had suffered a severe head injury, and all three required insertion of a ventriculoperitoneal shunt. GCS Glasgow Coma Scale, $P T H$ post-traumatic hydrocephalus 
Table 1 List of clinical radiological features present on admission to hospital and on initial CT head scans of paediatric patients following severe TBI (GCS 8 or lower on admission)

\begin{tabular}{lll}
\hline Clinical/radiological feature & No PTH $(n=26)$ & PTH $(\mathrm{n}=3)$ \\
\hline Emergency craniotomy/craniectomy & $16(61 \%)$ & $1(33 \%)$ \\
Other emergency operation & $17(65 \%)$ & $0(0 \%)$ \\
Ventriculomegaly on initial scan & $0(0 \%)$ & $0(0 \%)$ \\
IVH & $3(12 \%)$ & $3(100 \%)$ \\
SAH & $7(27 \%)$ & $3(100 \%)$ \\
IVH + SAH & $2(8 \%)$ & $3(100 \%)$ \\
Skull fracture & $13(50 \%)$ & $3(100 \%)$ \\
SDH & $10(39 \%)$ & $3(100 \%)$ \\
EDH & $8(31 \%)$ & $1(33 \%)$ \\
Cortical contusions & $4(15 \%)$ & $0(0 \%)$ \\
Midline shift & $3(12 \%)$ & $1(33 \%)$ \\
Effacement of basal cisterns & $3(12 \%)$ & $0(0 \%)$ \\
Petechial haemorrhage & $3(12 \%)$ & $0(0 \%)$ \\
\hline
\end{tabular}

$I V H$ intraventricular haemorrhage, $S A H$ subarachnoid haemorrhage, $S D H$ subdural haematoma, EDH extradural haematoma, GCS Glasgow Coma Scale

Rumalla et al. also reviewed clinical pathologies that were shown to be associated with PTH, which included SAH and SDH [10]. The present study also identified that these features were either significantly associated with PTH (SAH) or demonstrated a trend towards an association (SDH). The focus of the present study was to review radiological features which may raise the probability of PTH in children, which was not the focus of the two larger studies $[5,10]$.

One explanation for the prevalence rates identified in the present study is that, to our knowledge, this is one of the first studies in which there has been a focused assessment of the prevalence of PTH in children admitted to a single paediatric

Table 2 Radiological features associated with PTH in the study cohort

\begin{tabular}{lllll}
\hline Radiological feature & No of patients & PTH & No PTH & $p$ value \\
\hline IVH & 6 & 3 & 3 & 0.05 \\
SAH & 10 & 3 & 7 & 0.03 \\
SAH + IVH & 5 & 3 & 2 & $<0.01$ \\
SDH & 13 & 3 & 10 & 0.07 \\
Skull fracture & 16 & 3 & 13 & 0.2 \\
Effacement of BS & 2 & 0 & 2 & 1 \\
Midline shift & 4 & 1 & 3 & 1
\end{tabular}

All three patients with PTH suffered a skull fracture, IVH, SAH, and SDH. The association between PTH and initial IVH and SAH (particularly when both were present together) reached statistical significance. Fisher's exact test was used for statistical analysis, with $p$ values $<0.05$ being considered significant

$B S$ basal cisterns neurosurgical centre only. The two previous, albeit larger, studies pooled patients from a wide range of hospitals and primary care settings $[5,10]$. This may have diluted the prevalence of the condition, with large numbers of mild and moderate TBI patients masking a higher prevalence of PTH amongst children with more severe brain injuries who are admitted directly to neurosurgery units. Our study and others have purported that severe TBI patients are the group most likely to develop PTH [4]. Furthermore, the two larger studies of PTH in children thus far defined paediatric populations as $<21$ years old, which is an older cut-off compared with that used in the UK $(<16$ years old). The average age of patients in these large cross-sectional studies was thus older than in the present work (12 years vs 7 years). Finally, the cross-sectional population-based studies provide snapshot, retrospective data, with short follow-up periods of around 12 months $[5,8,10]$. The authors of one of the studies remind readers that their databases are only reliable for reviewing inpatient stays, complications, and outcomes, meaning that patients who developed PTH after being discharged from their initial hospital admission will not have been captured using their methodology [10]. Our study group was a smaller, but focused cohort of children admitted to a neurosurgical unit. Our longer median follow-up data (median 48 months) helps support the notion that PTH is amenable to treatment by VP shunting and corroborates other studies' inferences that long-term outcomes are improved for PTH patients by CSF diversion [7, 13].

In adult patients, the factors that have been associated with the development of PTH include younger age [7, 13, 14], the presence of subdural haematoma $[8,10]$, and the need for decompressive craniectomy [7]. However, these factors have not been consistently demonstrated in all case series, with some even reporting opposite associations, such as one study which identified older age as a risk factor for PTH [9]. Nonetheless, the factors identified in this study that may increase clinical suspicion of subsequent development of PTH are severity of TBI, IVH, SAH, and the combination of IVH and SAH together. There was also a trend towards an association between subdural haematoma and a higher probability of PTH. All these proposed risk factors have been identified in other works in the adult and paediatric PTH literature $[3,5,7,8,10,15$, 16]. These seem reasonable factors to predict $\mathrm{PTH}$, given that injury severity and the volume of intracranial blood load is associated with the development of hydrocephalus in other pathologies, such as non-traumatic subarachnoid haemorrhage [3].

Our study did not identify emergency neurosurgery, such as craniectomy, as increasing the probability of PTH. However, decompressive craniectomy (DC) has been associated with up to a $50 \%$ risk of development of PTH in adults [6-8]. The risk of PTH is even higher in adult DC patients who also have or develop subdural haematomas/hygromas [8]. The small sample size of the present study may explain why we did not identify DC as a risk factor in our study 
Table 3 Clinical features, radiological details, and clinical outcomes for all patients who developed PTH in the study cohort

\begin{tabular}{|c|c|c|c|c|c|c|c|}
\hline Age & Skull fracture & SDH size & SDH location & $\begin{array}{l}\text { Interval } \\
\text { (injury to PTH) }\end{array}$ & Other surgery & CSF diversion & eGOS \\
\hline \multirow[t]{2}{*}{9 years } & $\begin{array}{l}\text { Left frontal bone } \\
\text { Left occipital bone }\end{array}$ & \multirow[t]{2}{*}{$4 \mathrm{~mm}$} & \multirow[t]{2}{*}{$\begin{array}{l}\text { Left temporal }+ \\
\quad \text { interhemispheric }\end{array}$} & \multirow[t]{2}{*}{1 month } & \multirow[t]{2}{*}{ Nil } & \multirow[t]{2}{*}{ VP shunt (0 revisions) } & \multirow[t]{2}{*}{8} \\
\hline & Right parietal bone (non-depressed) & & & & & & \\
\hline 9 years & $\begin{array}{l}\text { Left temporal bone } \\
\text { Left occipital bone (non-depressed) }\end{array}$ & $12 \mathrm{~mm}$ & Left convexity & 1 month & Craniotomy & VP shunt (0 revisions) & 5 \\
\hline \multirow[t]{2}{*}{10 months } & $\begin{array}{l}\text { Left occipital bone } \\
\text { Right temporal bone }\end{array}$ & \multirow[t]{2}{*}{$4 \mathrm{~mm}$} & \multirow[t]{2}{*}{$\begin{array}{l}\text { Right temporal }+ \\
\quad \text { adjacent to torcula }\end{array}$} & \multirow[t]{2}{*}{3 months } & \multirow[t]{2}{*}{ Nil } & \multirow[t]{2}{*}{ VP shunt (0 revisions) } & \multirow[t]{2}{*}{6} \\
\hline & Right occipital bone (non-depressed) & & & & & & \\
\hline
\end{tabular}

None of this subcohort suffered a depressed skull fracture. The interval between injury and the development of PTH was between 1 and 3 months postTBI. One patient underwent emergency surgery prior to their VP shunt insertion. No patients have required revision of their VP shunts, and eGOS ranged between 5 and 8

eGOS Extended Glasgow Outcome Scores, VP shunt ventriculoperitoneal shunt

population; only three patients required a DC. As a single paediatric neurosurgery centre, we are unlikely to improve the power of future studies in a timely manner with our current patient workload. Therefore, the most efficient way to maximize the power of much-needed follow-up studies, without diluting study populations with non-neurosurgical unit data and avoiding the snapshot nature of cross-sectional methodologies, will be to design a multicentre, prospectively maintained national database of paediatric TBI. This database could cover admissions, management, and complications of children who have suffered a head injury severe enough to warrant referral or admission to a neurosurgical unit throughout the country. Such a database could drastically improve the ability of clinicians to mine valuable data to investigate prognostic factors and guide management of paediatric TBI. This would include, but not necessarily be limited to, formally defining the predictive factors and optimal treatment strategies for paediatric PTH.

\section{Conclusion}

The prevalence of PTH in this study's neurosurgical paediatric population was $2.7 \%$, which is higher than that in previous studies of paediatric TBI. Potential risk factors which may increase the probability of the development of PTH in children may include low GCS (i.e. severity of TBI) and the presence of intracranial blood load in the form of IVH, SAH, and possibly SDH. A larger cohort of paediatric TBI patients admitted to neurosurgery should be analysed to elucidate predictive factors for PTH. We propose a national, prospective, multicentre evaluation of paediatric PTH prevalence, presentation, risk factors, and management. This evidence could inform the contemporary management and improve the clinical outcomes of children with PTH.
Acknowledgements We acknowledge the assistance of the paediatric and neurosurgery clinical departments at the UHW for their assistance in data collection logistics.

Author contribution All authors contributed to the study conception and design. Material collection, data collection, and analysis were performed by Ronak Ved, Rebecca Fraser, and Sarah Hamadneh, and all authors reviewed and contributed to previous versions of the manuscript. All authors read and approved the final manuscript.

Data availability The datasets generated during and/or analysed during the current study are available from the corresponding author on reasonable request.

Compliance with ethical standards All procedures performed in studies involving human participants were in accordance with the ethical standards of the Cardiff and Vale University Health Board research committee and with the 1964 Helsinki declaration and its later amendments.

Conflict of interest On behalf of all authors, the corresponding author states that there are no conflicts of interest regarding this manuscript.

Informed consent Informed consent was obtained from any individual participants and/or their next of kin for their anonymous data to be included in the database from which the data was derived for this study.

Open Access This article is licensed under a Creative Commons Attribution 4.0 International License, which permits use, sharing, adaptation, distribution and reproduction in any medium or format, as long as you give appropriate credit to the original author(s) and the source, provide a link to the Creative Commons licence, and indicate if changes were made. The images or other third party material in this article are included in the article's Creative Commons licence, unless indicated otherwise in a credit line to the material. If material is not included in the article's Creative Commons licence and your intended use is not permitted by statutory regulation or exceeds the permitted use, you will need to obtain permission directly from the copyright holder. To view a copy of this licence, visit http://creativecommons.org/licenses/by/4.0/. 


\section{References}

1. Beyerl B, Black PM (1984) Posttraumatic hydrocephalus. Neurosurgery 15(2):257-261 Retrieved from https://www.ncbi. nlm.nih.gov/pubmed/6384812

2. Dewan MC, Rattani A, Gupta S et al (n.d.) Estimating the global incidence of traumatic brain injury. J Neurosurg 1:1-18 Retrieved from https://www.ncbi.nlm.nih.gov/pubmed/29701556

3. Tian HL, Xu T, Hu J, Cui YH, Chen H, Zhou LF (2008) Risk factors related to hydrocephalus after traumatic subarachnoid hemorrhage. Surg Neurol 69:241-246

4. Matsumoto S (1990) Post-traumatic hydrocephalus in children: pathophysiology and classification. Annu Rev Hydrocephalus 6: 147-148 Retrieved from https://link.springer.com/chapter/10. 1007/978-3-662-11152-9 107

5. Bonow RH, Oron AP, Hanak BW, Browd SR, Chesnut RM, Ellenbogen RG, Vavilala MS, Rivara FP (2018) Post-traumatic hydrocephalus in children: a retrospective study in 42 pediatric hospitals using the pediatric health information system. Neurosurgery 83(4):732-739

6. De Bonis P, Pompucci A, Mangiola A et al (2010) Post-traumatic hydrocephalus after decompressive craniectomy: an underestimated risk factor. J Neurotrauma 27(11):1965-1970

7. Low CY, Low YY, Lee KK et al (2013) Post-traumatic hydrocephalus after ventricular shunt placement in a Singaporean neurosurgical unit. J Clin Neurosci 20(6):867-872

8. Vedantam A, Yamal JM, Hwang H, Robertson CS, Gopinath SP (2018) Factors associated with shunt-dependent hydrocephalus after decompressive craniectomy for traumatic brain injury. $\mathrm{J}$ Neurosurg 128:1547-1552

9. Jiao QF, Liu Z, Li S, Zhou LX, Li SZ, Tian W, You C (2007) Influencing factors for posttraumatic hydrocephalus in patients suffering from severe traumatic brain injuries. Chin J Traumatol 10:159-162

10. Rumalla K, Letchuman V, Smith KA, Arnold PM (2018) Hydrocephalus in pediatric traumatic brain injury: national Incidence, risk factors, and outcomes in 124,444 hospitalized patients. Pediatr Neurol 80:70-76

11. Giza CC, Mink RB, Madikians A (2007) Pediatric traumatic brain injury: not just little adults. Curr Opin Crit Care 13(2):143-152 Retrieved from: https://www.ncbi.nlm.nih.gov/pubmed/17327734

12. Gudeman SK, Kishore PR, Becker DP, Lipper MH, Girevendulis AK, Jeffries BF, Butterworth JF 4th (1981) Computed tomography in the evaluation of incidence and significance of post-traumatic hydrocephalus. Radiology 141:397-402

13. Silver B, Chinarian J (1997) Neurologic improvement following shunt placement for post-traumatic hydrocephalus in a child. Pediatr Rehabil 1(2):123-126

14. Figaji AA (2017) Anatomical and physiological differences between children and adults relevant to traumatic brain injury and the implications for clinical assessment and care. Front Neurol 8: 685 Retrieved from https://www.ncbi.nlm.nih.gov/pmc/articles/ PMC5735372/

15. Licata C, Cristofori L, Gambin R, Vivenza C, Turazzi S (2001) Post-traumatic hydrocephalus. J Neurosurg Sci 45(3):141-149 Retrieved from https://www.ncbi.nlm.nih.gov/pubmed/11731738

16. Mazzini L, Campini R, Angelino E, Rognone F, Pastore I, Oliveri G (2003) Posttraumatic hydrocephalus: a clinical, neuroradiologic, and neuropsychologic assessment of long-term outcome. Arch Phys Med Rehabil 84:1637-1641

Publisher's note Springer Nature remains neutral with regard to jurisdictional claims in published maps and institutional affiliations. 